\title{
Die Coulomb-Energien leichter Atomkerne
}

\author{
Von \\ J. JÄNECKE * \\ Mit 5 Figuren im Text \\ (Eingegangen am 4. Juli 1960)
}

A compilation of the known data on Coulomb energy differences of isobaric doublets and isobaric triplets is given. Plots of the Coulomb energy differences versus $\bar{Z} / A^{\frac{1}{3}}$ with $\bar{Z}=\left(Z_{1}+Z_{2}\right) / 2$ show an analogous shell structure behaviour for the three series with $2 \bar{Z}=A-1, A$ and $A+1 \quad\left(T=1, \frac{1}{2}\right.$ and 1), i.e. discontinuities at the closed shells at $A=4,16$ and 40 and the closed subshell at $A=32$ and oscillations mainly being due to Coulomb proton-proton pairing energy. A positive energy shift of the lowest states with $T=1$ of all self-conjugate nuclei with $A=4 n+2$ seems to be indicated by the experimental data. A semi-empirical formula is given that describes the data.

\section{Einleitung}

Aus der Lage der analogen Zustände von Isobarenmultipletts lassen sich bei den leichten Atomkernen Aussagen über die Coulomb-Energien. gewinnen. Beispielsweise kann man die Coulomb-Energiedifferenzen der Spiegelkerne aus deren Zerfallsenergien bestimmen. Mit dem Verhalten dieser Coulomb-Energiedifferenzen zwischen den Grundzuständen zum Isobarenspin $T=\frac{1}{2}$ befassen sich eine Reihe von Arbeiten ${ }^{1-18}$. Die

* Jetzt: The University of Michigan, Ann Arbor (U.S.A.).

1 Bethe, H. A.: Phys. Rev. 54, 436 (1938).

2 Wigner, E.P.: Phys. Rev. 56, 519 (1939).

${ }^{3}$ White, M. G., E.C. Creutz, L.A. Delsasso u. R. R. Wilson: Phys. Rev. 59, 63 (1941).

4 Feenberg, E., u. G. Goertzel: Phys. Rev. 70, 597 (1946).

5 Cooper, L. N., u. E.M. Henley: Phys. Rev. 92, 801 (1953).

${ }^{6}$ Stähelin, P.: Helv, phys. Acta 26, 691 (1953).

7 Jancovici, B. G.: Phys. Rev. 95, 389 (1954).

8 Peaslee, D.C.: Phys. Rev. 95, 717 (1954).

9 Carlson, B.C., u. I. Talmi: Phys. Rev. 96, 436 (1954).

10 Scotr, J.M.C.: Progr. Nuclear Phys. 5, 172 (1956).

11 Kofoed-Hansen, O.: Nuclear Phys. 2, 441 (1956/57).

12 Sood, P.C., u. A.E.S. Green: Nuclear Phys. 4, 274 (1957).

${ }^{13}$ Welch, J.A., u. R. Wallace: Bull. Amer. Phys. Soc. II 3, 206 (1958). --

Wallace, R., u. J.A. Welch: Phys. Rev. 117, 1297 (1960).

14 Arnell, S.E., J. Dubois u. O. Almén: Nuclear Phys. 6, 196 (1958).

15 Swamy, N.V.V., u. A.S. Green: Phys. Rev. 112, 1719 (1958).

16 Kofoed-Hansen, O.: Rev. Mod. Phys. 30, 449 (1958).

17 UnNa, I.: Nuclear Phys. 8, 468 (1958).

${ }^{18}$ Cherry, R. D.: Phys. Rev. 115, 1243 (1959). 
Unstetigkeiten im Verlauf der Energiedifferenzen in Abhängigkeit von $A$ bei den doppelt magischen Zahlen sowie die überlagerten Strukturen lassen sich theoretisch qualitativ beschreiben. Weit weniger ist jedoch über die Coulomb-Energiedifferenzen zwischen den tiefsten Zuständen zum Isobarenspin $T=1$ bekannt $6,8,11,16,19,20,21$. Ziel dieser Arbeit ist es, aus einer Zusammenstellung aller verfügbaren Daten eventuelle Gemeinsamkeiten im Verhalten der beiden Reihen von Coulomb-Energiedifferenzen $\mathrm{zu} T=1$ mit der Reihe $\mathrm{zu} T=\frac{1}{2}$ (Spiegelkerne) zu erkennen und eine gemeinsame Beschreibung zu finden.

\section{Die ersten und zweiten Coulomb-Energiedifferenzen}

Die Werte für die ersten Coulomb-Energiedifferenzen ${ }^{16}$

$$
1 \Delta(\bar{Z}, \bar{N}, A)=E_{c}\left(\bar{Z}+\frac{1}{2}, \bar{N}-\frac{1}{2}, A\right)-E_{c}\left(\bar{Z}-\frac{1}{2}, \bar{N}+\frac{1}{2}, A\right)
$$

mit $\bar{Z}=\left(Z_{1}+Z_{2}\right) / 2$ und $\bar{N}=\left(N_{1}+N_{2}\right) / 2$ (Index 1 bzw. 2 bezieht sich auf Anfangs- und Endkern) ergeben sich aus den gemessenen maximalen $\beta$-Energien oder aus $Q$-Werten von $(p, n)-,(\bar{d}, n)-,(d, p)-,(\alpha, n)$ - und $(\alpha, p)$-Reaktionen nach den folgenden Beziehungen:

$$
\begin{aligned}
& { }^{1} \Delta=1,804+E_{\beta^{+}}, \\
& { }^{1} \Delta=0,782+E_{K}, \\
& 1 \Delta=0,782-E_{\beta^{-}}, \\
& 1 \Delta=-Q_{p n}, \\
& 1 \Delta=Q_{d p}-Q_{d n}, \\
& 1 \Delta=Q_{\alpha p}-Q_{\alpha n},
\end{aligned}
$$

Die Beziehungen (2a) bis (2c) gelten für $\beta$-Übergänge bzw. $K$-Einfang zwischen analogen Zuständen. Gl. (2d) gilt, wenn die Zustände von Anfangs- und Endkern analog sind. Gl. (2e) und (2f) gelten, wenn die Reaktionen vom gleichen Kern ausgehen und zu analogen Zuständen der Endkerne führen.

Sämtliche Gln. (2a) bis (2f) lassen sich auf eine andere Klasse von Zerfällen und Reaktionen erweitern, wenn ein selbstkonjugierter Kern $(Z=N=A / 2)$ beteiligt ist. Geht nämlich der Zerfall oder die Reaktion über den tiefsten Zustand zum Isobarenspin $T=0$ und nicht über den tiefsten Zustand zu $T=1$, so bleiben die Beziehungen guiltig, wenn man auf der rechten Seite $\pm \Delta_{10}$ addiert. $\Delta_{10}$ ist der Energieabstand zwischen den tiefsten Zuständen zu $T=1$ und $T=0$. Das positive Zeichen gilt für

19 Moszkowsky, S.A., .. D.C. Peaslee: Phys. Rev. 93, 455 (1954).

20 Wilkinson, D.H.: Phil. Mag. 1, 1031 (1956).

21 Temmer, G.M., u. N.P. Heydenburg: Phys. Rev. 111, 1303 (1958). 
Zerfälle oder Reaktionen zwischen dem selbstkonjugierten und dem neutronenreichen Kern des Isobarentripletts, d.h. für $T_{z}=0 \leftrightarrow T_{z}=+1$, das negative Zeichen gilt entsprechend für den protonenreichen und den selbstkonjugierten Kern des Isobarentripletts, d.h. für $T_{z}=-1 \leftrightarrow T_{z}=0$.

In Tabelle 1 sind sämtliche gemessenen Coulomb-Energiedifferenzen zusammengestellt ${ }^{22}$. Spalte 3 enthält die Werte ${ }^{1} \Delta$ für $T=\frac{1}{2}$ (Spiegelkerne), Spalte 2 und 4 die Werte für $T=1$. Die Energiewerte $\Delta_{10}$ in Spalte 5 geben die Lage des ersten Zustandes zum Isobarenspin $T=1$ an.

Die Coulomb-Energiedifferenzen werden unter der Annahme einer homogenen Ladungsverteilung im Kern durch folgende Beziehung beschrieben ${ }^{16}$ :

$$
{ }^{1} \Delta(\bar{Z}, \bar{N}, A)=1,2 \cdot \frac{e^{2}}{r_{0}(\bar{A})} \cdot \frac{\bar{Z}}{A^{\frac{1}{3}}}-S_{p} .
$$

Dabei ist $r_{0}(A) \cdot A^{\frac{T}{3}}$ der Kernradius mit einem langsam mit $A$ veränderlichen Äqivalenzradius $\gamma_{0}(A) ; \bar{Z}$ steht für $\left(Z_{1}+Z_{2}\right) / 2$ und $S_{p}$ ist die Coulomb-Selbstenergie ${ }^{5,8,10,15,18}$ des Protons. Es ist nach Gl. (3) daher vernünftig, die Werte aus Tabelle 1 , Spalte 2 bis 4 als Funktion von $\bar{Z} / A^{\frac{1}{3}}$ aufzutragen. Fig. 1 zeigt die drei Kurven für $T=\frac{1}{2}, \bar{Z}=A / 2$, für $T=1, \bar{Z}=(A-1) / 2$ und für $T=1, \bar{Z}=(A+1) / 2$. Man erkennt in den drei Darstellungen Unstetigkeiten bei den abgeschlossenen Hauptschalen bei $A=4,16$ und 40 sowie schwach bei der abgeschlossenen Unterschale bei $A=32$. Außerdem überlagern sich Oszillationen, deren Amplitude bei den Isobarentripletts kleiner ist als bei den Isobarendubletts. Die eingezeichneten Geraden werden später besprochen.

Die Diskussion der überlagerten Strukturen wird erleichtert durch Bildung der zweiten Coulomb-Energiedifferenzen:

$$
\begin{aligned}
& { }^{2} \Delta\left(\bar{Z}+\frac{1}{2}, \bar{N}+\frac{1}{2}, A+1\right)=1 \Delta(\bar{Z}+1, \bar{N}+1, A+2)-1 \Delta(\bar{Z}, \bar{N}, A) \\
& { }^{2} \Delta^{n}\left(\bar{Z}, \bar{N}+\frac{1}{2}, A+\frac{1}{2}\right) \quad{ }^{1} \Delta(\bar{Z}, \bar{N}+1, A+1) \quad-1 \Delta(\bar{Z}, \bar{N}, A) \\
& { }^{2} \Delta^{p}\left(\bar{Z}+\frac{1}{2}, \bar{N}, A+\frac{1}{2}\right) \quad=1 \Delta(\bar{Z}+1, \bar{N}, A+1) \quad-1 \Delta(\bar{Z}, \bar{N}, A)
\end{aligned}
$$

${ }^{2} \Delta$ gibt an, in welcher Weise sich die Coulomb-Energiedifferenzen unterscheiden, wenn die Anfangs- und Endkerne jeweils ein Proton und ein Neutron mehr enthalten. Entsprechend beschreiben ${ }^{2} \Delta^{n}$ und ${ }^{2} \Delta^{p}$ die Änderungen beim Hinzufügen eines Neutrons oder Protons allein. Man gewinnt diese Werte aus Tabelle 1 durch Differenzbildung vertikal oder diagonal benachbarter Werte in den Spalten 2, 3 und 4 .

22 Werte nach F.AJzenberg-Selove u. T. LAuritsen: Nuclear Phys. 11, 1 (1959); Endt, P.M., u. C.M. Braams: Rev. Mod. Phys. 29, 683 (1957); Miller, J.H., u. D.C. Sutron: Bull. Amer. Phys. Soc. II 3, 206 (1958); Hashrmoto, Y., u. W. P. Alford: Phys. Rev. 116, 981 (1959), und den Zitaten 13, 21, 25 und 27. 
Tabelle 1. Erste Coulomb-Energiedifferenzen $1 \Delta$ zwischen Isobarendubletts $\left(T=\frac{1}{2}\right)$ und Isobarentripletts $(T=1)$ sowie Energieunterschied $A_{10}$ der tiefsten Zustände zu $T=1$ und $T=0$

\begin{tabular}{|c|c|c|c|c|c|c|c|}
\hline (1) & (2) & $\begin{array}{c}\text { (3) } \\
{ }^{2} \Delta \mathrm{in} \mathrm{MeV}\end{array}$ & (4) & $\begin{array}{c}\text { (5) } \\
\Delta_{10} \text { in } \mathrm{MeV}\end{array}$ & (6) & $\begin{array}{c}\overline{(7)} \\
\overline{Z_{1}} / A^{\frac{1}{3}}\end{array}$ & (8) \\
\hline$A$ & $\begin{aligned} 2 \bar{Z} & =A-1 \\
A & =4 n ; 4 n+2 \\
T & =1 \\
T_{z} & =0 \leftrightarrow T_{z}=+1\end{aligned}$ & $\begin{aligned} 2 \bar{Z} & =A \\
A & =4 n+1 ; 4 n+3 \\
T & =\frac{1}{2} \\
T_{z} & =-\frac{1}{2} \leftrightarrow T_{z}=+\frac{1}{2}\end{aligned}$ & $\begin{aligned} 2 \bar{Z} & =A+1 \\
A & =4 n ; 4 n+2 \\
T & =1 \\
T_{z} & =-1 \leftrightarrow T_{z}=0\end{aligned}$ & $\begin{aligned} 2 Z & =A \\
A & =4 n ; 4 n+2 \\
T & =1 \leftrightarrow T=0 \\
T_{z} & =0\end{aligned}$ & 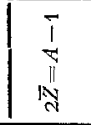 & II & $\underset{\substack{\text { I } \\
\text { II }}}{\text { II }}$ \\
\hline 1 & & 0,000 & & & & 00 & \\
\hline 2 & & & & & 0,397 & & 1,190 \\
\hline $\begin{array}{l}3 \\
4\end{array}$ & & $0,764 \pm 0,001$ & & & 0.045 & 1,040 & \\
\hline 5 & & $0,839 \pm 0,050$ & & & 0,945 & 1,462 & 1,575 \\
\hline $\begin{array}{l}6 \\
7\end{array}$ & $0,810 \pm 0,020$ & $1644^{\circ}+2$ & $1,660 \pm 0,200$ & $3,560 \pm 0,006$ & 1,375 & & 1,926 \\
\hline 8 & $1,470 \pm 0,050$ & $1,045]$ & $2,090 \pm 0,010$ & $16,670 \pm 0,010$ & 1,750 & 1,830 & 2,250 \\
\hline $\begin{array}{r}9 \\
10\end{array}$ & $1,966+0,007$ & $1,852 \pm 0,002$ & & & & 2,163 & \\
\hline 11 & 1,9 & $2,763 \pm 0,003$ & 90 & $1,739 \pm 0,005$ & 2,088 & 2,473 & 2,552 \\
\hline $\begin{array}{l}12 \\
13\end{array}$ & $2,520 \pm 0,020$ & $3,007 \pm$ & $3,130 \pm 0,080$ & $15,110 \pm 0,010$ & 2,402 & 2.764 & 2,838 \\
\hline 14 & $2,940 \pm 0,002$ & & $3,614 \pm 0,008$ & $2,312 \pm 0,001$ & 2,697 & & 3,111 \\
\hline $\begin{array}{l}15 \\
16\end{array}$ & $3,160 \pm 0$ & $3,543 \pm 0,002$ & $3,630 \pm 0,030$ & $12,780 \pm 0,010$ & 2.976 & 3,041 & 3.373 \\
\hline 17 & & $3,551 \pm 0,006$ & & $12,700 \pm 0,010$ & & 3,306 & \\
\hline 18 & $3,530 \pm 0,010$ & $4025+2+1$ & $3,920 \pm 0,200$ & $1,085 \pm 0,010$ & 3,243 & & 3,624 \\
\hline $\begin{array}{l}19 \\
20\end{array}$ & $\{3,700 \pm 0,100$ & $4,025 \pm 0,004$ & $\{6,150 \pm 0,200$ & $\left\{\begin{array}{l}9,970 \pm 0,100 \\
0\end{array}\right.$ & & 3,560 & 3.868 \\
\hline 21 & $\{4,34$ & $4,310 \pm 0,020$ & $\lfloor 5,510 \pm 0,200$ & $\{10,670 \pm$ & 3,499 & 3,806 & 3,868 \\
\hline 22 & $4,289 \pm 0,005$ & $4.844+5=$ & & $0,666 \pm 0,004$ & 3,748 & & 4,103 \\
\hline 24 & $4,740 \pm 0,100$ & & $5,300 \pm 0,300$ & $9,470+0,100$ & 3,987 & 4,044 & 4.333 \\
\hline $25+5+2$ & & $5,080 \pm 0,020$ & & & & 4,274 & \\
\hline 26 & $5,006 \pm 0,010$ & 5,5 & & $0,228 \pm 0,005$ & 4,219 & -2 & 4,556 \\
\hline 28 & $5,510 \pm 0,100$ & & $5,230 \pm 0,500$ & $9,380 \pm 0,100$ & 4,446 & 4,500 & 4,774 \\
\hline $2 c-2+2=$ & $=730+0030$ & $5,748 \pm 0,005$ & & & & 4,720 & \\
\hline $\begin{array}{l}30 \\
31\end{array}$ & $5,730 \pm 0,020$ & $6,233+0$ & & $0,686 \pm 0,006$ & 4,666 & 4034 & 4,986 \\
\hline 32 & $6,060 \pm 0,060$ & & $6,710 \pm 0,400$ & $6,980 \pm 0,060$ & 4,882 & & 5,196 \\
\hline 33 & $6.304+0.0$ & $6,310 \pm 0,050$ & & $1-0$ & & 5,143 & \\
\hline $\begin{array}{l}34 \\
35\end{array}$ & $6,304 \pm 0,0$ & $6,760 \pm 0,040$ & & $-0,145 \pm 0,003$ & | 5,093 & 5,350 & 5,400 \\
\hline 36 & & Cono it & & & 5,299 & & 5,600 \\
\hline $\begin{array}{l}37 \\
38\end{array}$ & $6,820+0,050$ & $6,920 \pm 0,060$ & & $0.123+0.008$ & 5502 & 5,552 & $86>2+20$ \\
\hline 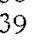 & & $7,280 \pm 0,030$ & & & & 5,750 & 5,800 \\
\hline $\begin{array}{l}40 \\
41\end{array}$ & $7,000 \pm 0,150$ & $\int 6,71$ & $7,210 \pm 0,400$ & $7,500 \pm 0,150$ & 5,701 & & 5,993 \\
\hline 19 & $6,600 \pm 0,900$ & 17 & & & 5,898 & 6,943 & 6,188 \\
\hline 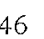 & & & & & & & \\
\hline J & & & & & & & $\begin{array}{l}0,559 \\
6,920\end{array}$ \\
\hline 54 & $9,070 \pm 0,100$ & & & & 7,010 & & 7,275 \\
\hline
\end{tabular}




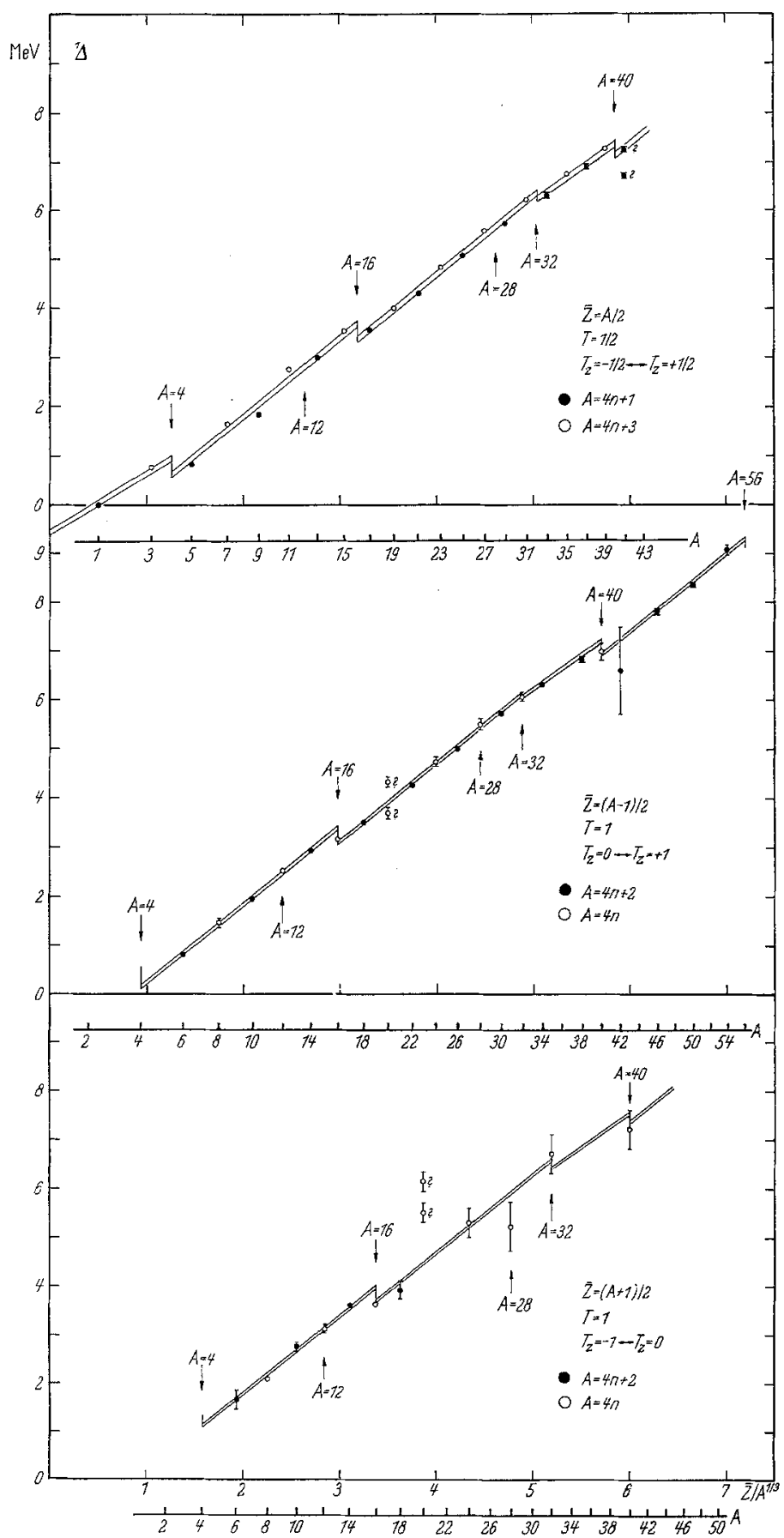

Fig. 1. Erste Coulomb-Energiedifferenzen ${ }^{1} \Delta$ bei $\bar{Z}=A / 2, T=\frac{1}{2}$, (Spiegelkerne), bei $\bar{Z}=(A-1) / 2, T=1$

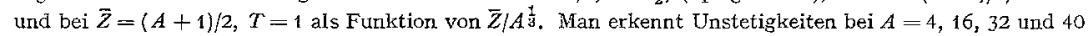
sowie überlagerte Strukturen. Die eingezeichneten Geraden werden durch Gl. (5) beschrieben 
Fig. 2 zeigt $2 \Delta$ für die drei einfachen Reihen (Differenzbildung in Spalte 3, 2 bzw. 4) sowie ${ }^{2} \Delta^{n}$ für die beiden kombinierten Reihen (Differenzbildung zwischen Spalte 2 und 3 bzw. 3 und 4) als Funktion von $A$. Auf die Darstellung von ${ }^{2} \Delta^{p}$ wurde verzichtet, da sich dort mehrere

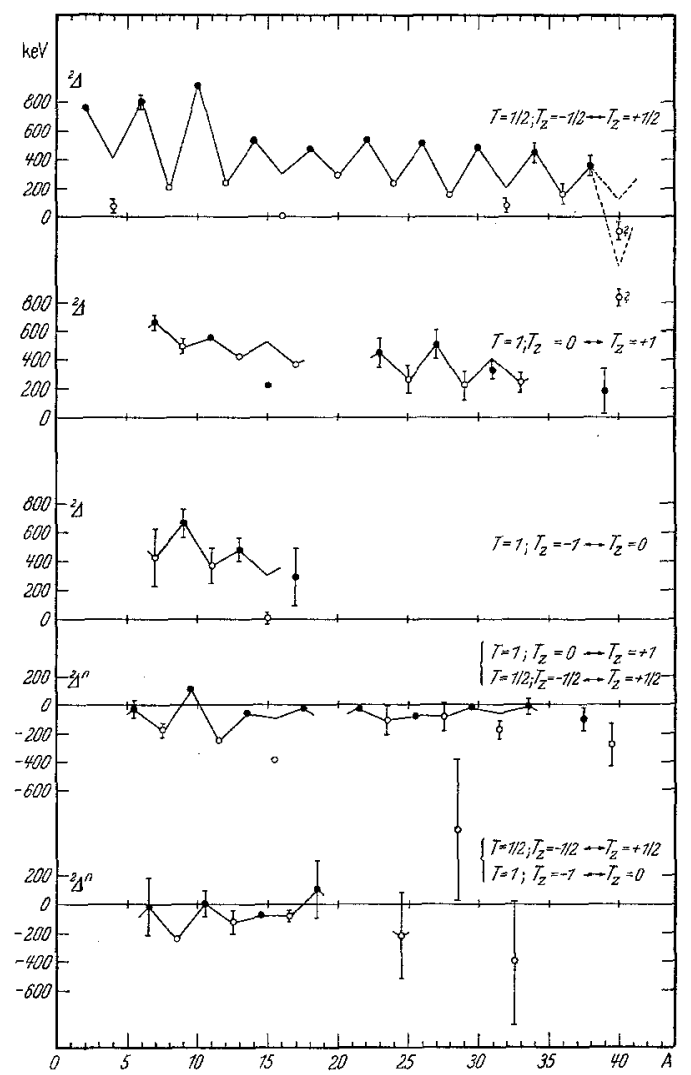

Fig. 2. Zweite Coulomb-Energiedifferenzen ${ }^{2} \Delta$ für die drei einfachen Reihen (Differenzbildung innerhalb der in der Figur angeführten Reihen von ${ }^{1} \Delta$ entsprechend Tabelle 1, Spalte 3,2 bzw. 4) und ${ }^{2} \Delta^{n}$ für die beiden kombinierten Reihen (Differenzbildung zwischen den in der Figur angeführten Reihen von ${ }^{1} \Delta$ entsprechend Tabelle 1, Spalten 2 und 3 bzw. 3 und 4) als Funktion von $A$. Die drei ersten Kurven entsprechen direkt Fig. 1. Die eingezeichneten. Kurven berücksichtigen die Unstetigkeiten von ${ }^{1} A$ bei den Schalenabschlüssen. Sie beschreiben dadurch nur noch die überlagerten Strukturen

Effekte überlagern. Die eingezeichneten Kurvenverläufe wurden bei $A=4,16,32$ und 40 um den jeweiligen Sprung in ${ }^{1} \Delta$ korrigiert. Die Kurven beschreiben dadurch im wesentlichen nur noch das Verhalten der überlagerten Strukturen. Die Amplituden der Oszillationen von ${ }^{2} \Delta$ sind in den beiden Kurven zu $T=1$ nur noch rund halb so groß wie die Amplituden bei $T=\frac{1}{2}$. AuBerdem deuten sich in ${ }^{2} \Lambda^{n}$ ebenfalls schwache Oszillationen an. 


\section{Diskussion}

Die Coulomb-Energiedifferenzen ${ }^{1} \Delta$ aus Tabelle 1 und Fig. 1 lassen sich zunächst rein empirisch in guter Näherung durch die folgende Gleichung beschreiben:

$$
{ }^{1} \Delta(\text { i. Schale })=E_{1}^{(i)} \cdot \frac{\bar{Z}}{A^{\frac{1}{3}}}-E_{2}^{(i)}+\delta \cdot E_{3}+\delta^{\prime} \cdot E_{4}
$$

mit $E_{1}^{(i)}, E_{2}^{(i)}$ nach Tabelle 2, Spalte 2 und 3

$$
\left.\begin{array}{l}
E_{3}=0,120 \mathrm{MeV} \\
E_{4}=0,060 \mathrm{MeV} \\
\left.2 \delta=1+(-1)^{\bar{Z}+1} \quad \text { (vgl. Tabelle 3, Spalte } 7 \text { bis } 9\right)
\end{array}\right\}
$$

\begin{tabular}{|c|c|c|c|}
\hline $\begin{array}{c}\text { (1) } \\
\text { Schale }\end{array}$ & $\begin{array}{c}(2) \\
E_{1}^{(i)}\end{array}$ & $\begin{array}{c}(3) \\
E_{2}^{(i)}\end{array}$ & $\begin{array}{l}(4) \\
y_{0}^{(i)}\end{array}$ \\
\hline$s \frac{1}{2}$ & $1,194 \mathrm{MeV}$ & $0,597 \mathrm{MeV}$ & $1,45 \cdot 10^{-13} \mathrm{~cm}$ \\
\hline$p \frac{3}{2}$ & 1,593 & 1,429 & 1,08 \\
\hline$p \frac{1}{2}$ & 1,593 & 1,429 & 1,08 \\
\hline$d \frac{5}{2}$ & 1,595 & 1,745 & 1,08 \\
\hline$s \frac{1}{2}$ & 1,595 & 1,745 & 1,08 \\
\hline$d \frac{3}{2}$ & 1,380 & 0,770 & 1,25 \\
\hline$f \frac{7}{2}$ & 1,600 & 2,280 & 1,08 \\
\hline
\end{tabular}

Die Energien $E_{1}^{(i)}, E_{2}^{(i)}, E_{3}$ und $E_{4}$ sind mittlere empirische Werte, die die experimentellen Daten am besten beschreiben. Durch den Anteil

Tabelle 2. Koeffizienten für Gl. (5)

$E_{1}^{(i)} \cdot \bar{Z} / A^{\frac{1}{3}}-E_{2}^{(i)}$ in Gl. (5) wird das Verhalten von ${ }^{1} \Delta$ mit den Unstetigkeiten an den abgeschlossenen Schalen bereits grob beschrieben. $E_{1}^{(i)}$ und $E_{2}^{(i)}$ sind mindestens innerhalb einer Unterschale konstant. Das additive Glied $\delta \cdot E_{3}+\delta^{\prime} \cdot E_{4}$ beschreibt dann die überlagerten Strukturen. Es nimmt die Werte 0,60 und $120 \mathrm{keV}$ an.

Die in Fig. 1 eingezeichneten Geraden folgen G1. (5) in Verbindung mit den Beziehungen (6) und Tabelle 2. Eine für die drei Reihen von Coulomb-Energiedifferenzen einheitliche Darstellung erhält man, wenn man von den experimentellen Werten, Energien von 0, 60 oder $120 \mathrm{keV}$ entsprechend dem Ausdruck $\delta \cdot E_{3}+\delta^{\prime} \cdot E_{4}$ abzieht. In Fig. 3 ist $1 A-$ $\left(\delta \cdot E_{3}+\delta^{\prime} \cdot E_{4}\right)$ als Funktion von $\bar{Z} / A^{\frac{1}{3}}$ aufgetragen. Die eingezeichneten Geraden werden durch $E_{1}^{(i)} \cdot \bar{Z} / A^{\frac{1}{3}}-E_{2}^{(i)}$ beschrieben. Die Übereinstimmung ist gut. Mit Ausnahme der Coulomb-Energiedifferenzen einiger Spiegelkerne in der $p 3 / 2$-Schale, insbesondere für $\mathrm{B}^{9}-\mathrm{Be}^{9}$ und 
$\mathrm{C}^{11}-\mathrm{B}^{11}$ sowie eventuell noch ${ }^{25}$ für $\mathrm{Sc}^{41}-\mathrm{Ca}^{41}$ ist die Übereinstimmung zwischen Gl. (5) und den experimentellen Werten besser als $50 \mathrm{keV}$.

Im folgenden sollen unter besonderer Berücksichtigung der Isobarentripletts die Effekte, die für das Zustandekommen von Gl. (5) verantwortlich sein könnten, diskutiert werden. Dies sind für die überlagerten

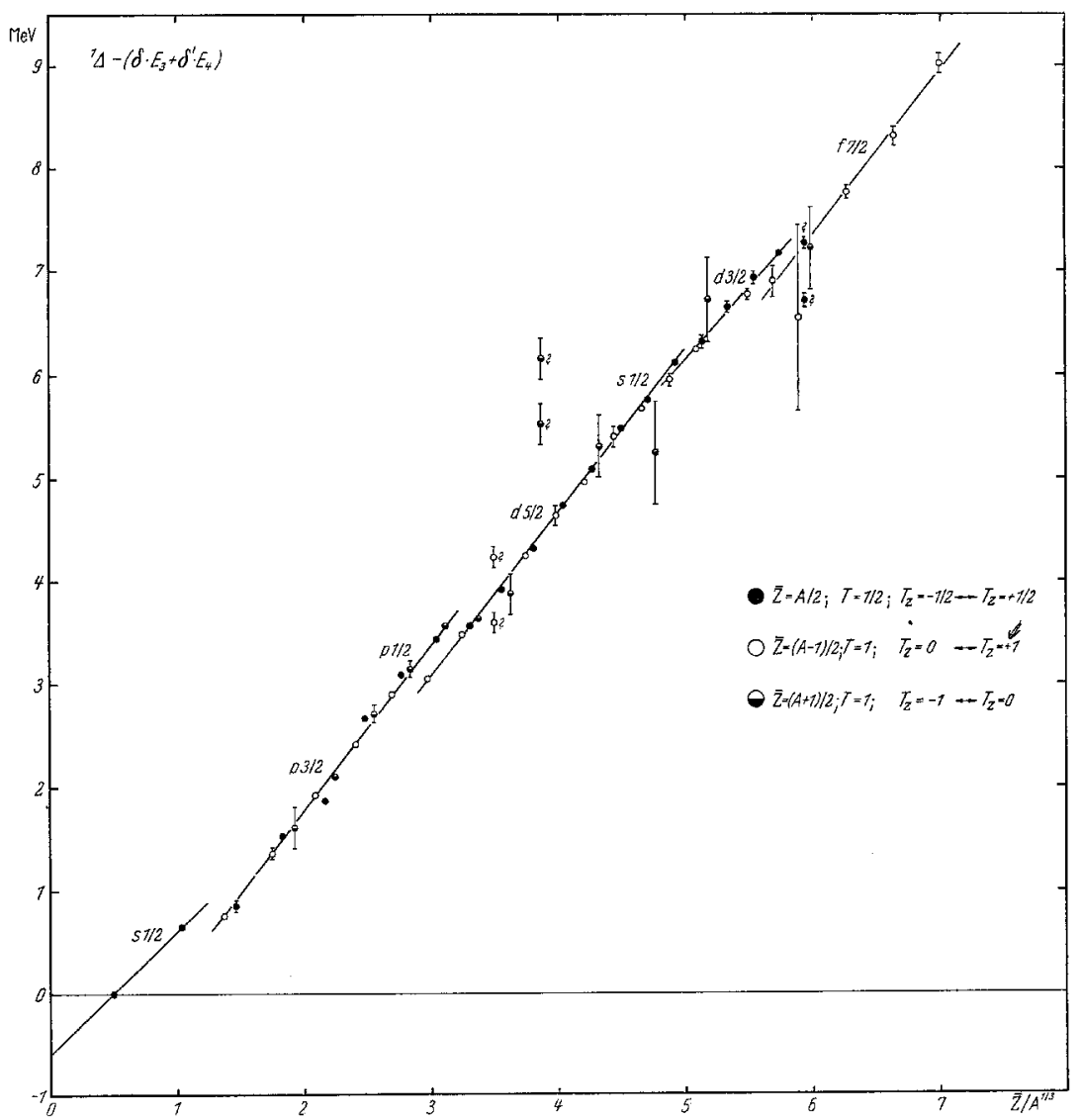

Fig. 3. ${ }^{1} \Delta-\left(\delta \cdot E_{3}+\delta^{\prime} \cdot E_{4}\right)$ als Funktion von $\bar{Z} / A$. Strukturen führen, lassen sich die drei Reihen von Coulomb-Energiedifferenzen (vg1. Fig. 1) durch gemeinsame Geraden beschreiben entsprechend Gl. (5)

Strukturen die Coulomb-Paarungsenergie $\left(E_{3}\right)$ und die Verschiebung des tiefsten $T=1$-Zustandes der selbstkonjugierten Kerne mit $A=4 n+2$ $\left(E_{4}\right)$. Es folgen die Fragen der Ladungsverteilung sowie die CoulombSelbstenergie des Protons $\left(E_{1}, E_{2}\right)$. Zum Abschluß werden die erwähnten Abweichungen diskutiert.

Es ist schon länger bekannt, daß der Verlauf der Coulomb-Energiedifferenzen der Spiegelkerne innerhalb einer Schale keinen glatten 
Verlauf zeigt. Vielmehr ist stets die Coulomb-Energiedifferenz der Kerne mit $A=4 n+3$ relativ größer als die der Kerne mit $A=4 n+1$. Dieser Effekt wird zurückgeführt auf eine zusätzliche Coulomb-Paarungsenergie zwischen abgesättigten Protonenpaaren 4,9,16,17. Während bei den Kernen mit $A=4 n+1$ die Zahl der abgesättigten Protonenpaare in Anfangs- und Endkern einander gleich ist, enthält bei $A=4 n+3$ der protonenreichere Kern stets ein abgesättigtes Paar mehr. Man weiß, daß gegenseitig abgesättigte Protonen einen kleineren mittleren Abstand haben. Hieraus ergibt sich dann die vergrößerte Coulomb-Energie des

Tabelle 3. Anzahl und Differenz der abgesättigten Protonenpaare

\begin{tabular}{|c|c|c|c|c|c|c|c|c|}
\hline (1) & (2) & (3) & (4) & (5) & (6) & (7) & (8) & (9) \\
\hline A & \multicolumn{5}{|c|}{ Anzahl abgesättigter Protonenpaare } & \multicolumn{3}{|c|}{$\begin{array}{l}\text { Differenz } \delta \text { abgesättigter } \\
\text { Protonenpaare }\end{array}$} \\
\hline & $\begin{array}{l}T=1 \\
T_{z}=+1\end{array}$ & $\begin{array}{r}\frac{1}{2} \\
+\frac{1}{2} \\
\end{array}$ & $\begin{array}{l}1 \\
0\end{array}$ & $\begin{array}{r}\frac{1}{2} \\
-\frac{1}{2} \\
\end{array}$ & $\begin{array}{r}1 \\
-1 \\
\end{array}$ & $\begin{array}{c}1 \\
+1 \leftrightarrow 0\end{array}$ & $+\frac{1}{2} \stackrel{\frac{1}{2}}{\leftrightarrow}-\frac{1}{2}$ & $\stackrel{1}{0 \leftrightarrow-1}$ \\
\hline $4 n$ & $n-1$ & $n$ & $n$ & $n$ & $n$ & 1 & 0 & 0 \\
\hline $\begin{array}{l}4 n+1 \\
4 n+2\end{array}$ & $n$ & $n$ & $n$ & $n$ & $n+1$ & 0 & U & 1 \\
\hline $\begin{array}{l}4 n+3 \\
4 n+4\end{array}$ & $n$ & $n$ & $n+1$ & $n+1$ & $n+1$ & 1 & 1 & 0 \\
\hline
\end{tabular}

betreffenden Kerns. Ein ähnliches Verhalten sollte auch für die Isobarentripletts zu erwarten sein. In Tabelle 3 sind die Anzahl solcher Protonenpaare sowie die jeweiligen Differenzen angegeben. Die Differenzen $\delta$ sind 0 oder 1. Sie werden durch G1. (6) beschrieben.

Bei einer angenähert konstanten Coulomb-Paarungsenergie $E_{3}$ sollten die drei Kurven für die ersten Differenzen ${ }^{1} \Delta$ in Fig. 1 überlagerte Oszillationen von gleicher Amplitude enthalten. Auch sollten alle zweiten Differenzen ${ }^{2} \Delta$ in Fig. 2 gleiche Oszillationen mit der doppelten CoulombPaarungsenergie als Amplitude und ${ }^{2} \Delta^{n}$ sollte keinerlei Strukturen aufweisen. Das tatsächliche in Abschnitt 2 beschriebene Verhalten entspricht sehr gut diesen Erwartungen mit einem $E_{3}$ von etwa $120 \mathrm{keV}$.

Eine genauere Analyse von ${ }^{1} \Delta$ und ${ }^{2} \Delta$ zeigt jedoch, da 3 sich bei den Isobarentripletts $(T=1)$ noch ein weiterer Effekt überlagert, der die Amplitude der Oszillationen verringert. Dieser Effekt äußert sich bei den Kernen mit $A=4 n+2$ in einer Erhöhung der Coulomb-Energiedifferenzen um etwa $60 \mathrm{keV}$ zwischen den Kernen mit $T_{z}=0 \leftrightarrow T_{z}=+1$ und $\bar{Z}=(A-1) / 2$ und einer entsprechenden Erniedrigung bei $T_{z}=-1 \leftrightarrow$ $T_{z}=0$ und $\bar{Z}=(A+1) / 2$. Dieses Verhalten wird formal durch den Ausdruck $\delta^{\prime} \cdot E_{4}$ in Gl. (5) beschrieben. Ohne diese Korrektur würden die Werte für $A=4 n+2$ in Fig. 3 systematische Abweichungen zeigen. Physikalisch bedeutet dieser zusätzliche Ausdruck, daß aus zunächst noch unbekannten Gründen der tiefste Zustand mit $T=1$ bei allen selbstkonjugierten Kernen mit $A=4 n+2$ offenbar um eine Energie von etwa 
$60 \mathrm{keV}$ angehoben ist. Bei den selbstkonjugierten Kernen mit $A=4 n$ ist dieser Effekt nicht vorhanden. In Fig. 4 sind die Einflüsse dieses Effektes und der Coulomb-Paarungsenergie auf die relative Lage der tiefsten analogen Zustände der Isobarendubletts und Isobarentripletts dargestellt. Die Energiedifferenzen zwischen den benachbarten analogen Zuständen werden durch $\delta \cdot E_{3}+\delta^{\prime} \cdot E_{4}$ wiedergegeben.

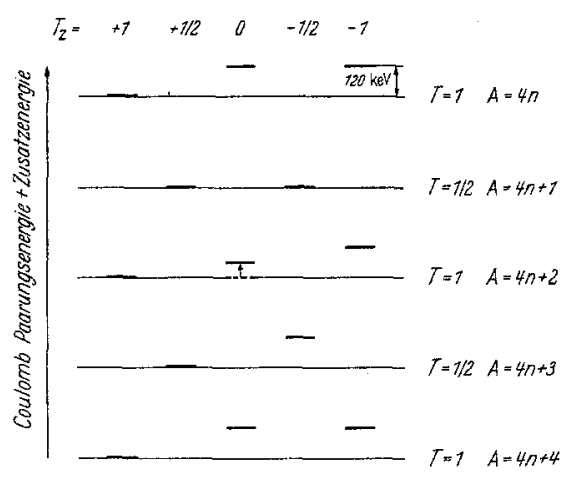

Fig. 4. Einfluß der Coulomb-Paarungsenergie und des zusätzlichen Effektes bei den selbstkonjugierten Kernen mit $A=4 n+2$ auf die relative Lage der analogen Grundzustände der Isobarendubletts und Isobarentripletts. Diese Energiebeiträge führen zu den iberlagerten Strukturen in Fig. 1 und Fig. 2. Die Energiedifferenzen zwischen benachbarten Zuständen betragen $\delta \cdot E_{3}+\delta^{\prime} \cdot E_{4}$
Bereits 1956 hat WILKINSON ${ }^{20}$ bei selbstkonjugierten Kernen nach einer Verschiebung der experimentell gemessenen tiefsten $T=1$-Zustände gegenüber einer berechneten Lage gesucht. Er fand einen mittleren Wert von etwa $-35 \mathrm{keV}$ durch Vergleich der experimentell bekannten tiefsten $T=1-\mathrm{Zu}-$ stände in diesen Kernen mit den experimentellen CoulombEnergiedifferenzen der benachbarten Spiegelkerne mit gleicher Protonenzahl. Diese Energien wurden mit dem Faktor $[(A-1) / A]^{\frac{1}{8}}$ multipliziert, um die Veränderung des Radius

durch die unterschiedlichen Neutronenzahlen zu berïcksichtigen. WALLACE und WELCH ${ }^{13}$ fanden neuerdings nach der gleichen Methode eine Verschiebung um $-(53 \pm 74) \mathrm{keV}$. Die Energiewerte von $-35 \mathrm{bzw}$. $-53 \mathrm{keV}$ sind nicht in Übereinstimmung mit den Ergebnissen dieser Arbeit.

Das beschriebene Verfahren soll nun etwas abgewandelt auf die Werte in Tabelle 1 angewendet werden. Die in Gl. (4) definierte und in Fig. 2 dargestellte Größe ${ }^{2} \Delta^{n}$ beschreibt bereits den Unterschied der fraglichen Coulomb-Energiedifferenzen. Man muß nur noch den Einfluß der bei unterschiedlichen Neutronenzahlen verschiedenen Radien berücksichtigen. Dies erreicht man entsprechend Gl. (5) durch Addition des Ausdrucks $E_{1}^{(i)} \cdot \Delta\left(\bar{Z} / A^{\frac{1}{3}}\right)$. Außerdem muß man wie in Fig. 2 an den Schalenabschlüssen die Unstetigkeiten von ${ }^{1} \Delta$ berücksichtigen. Dieses Verfahren berücksichtigt die Beeinflussung der Coulomb-Energie durch zusätzliche Neutronen aller Voraussicht nach besser als der oben angegebene Faktor $[(A-1) / A]^{\frac{1}{3}}$. Fig. 5 zeigt ${ }^{2} \Delta^{n}+E_{1}^{(i)} \cdot \Delta\left(\bar{Z} / A^{\frac{1}{3}}\right)$ als Funktion von $A$. Man sieht, daß in beiden Reihen die Werte, die die Kerne mit $A=4 n+2$ betreffen praktisch alle positiv sind, während die Werte für $A=4 n$ um die Energie 0 streuen. Bei den Punkten mit kleinem $A$ überlagern sich Unregelmäßigkeiten in den Coulomb-Energie- 
differenzen der Spiegelkerne. Die restlichen Punkte zeigen jedoch, daß für die Lage $\Delta_{10}$ (vgl. Abschn. 2 mit Tabelle 1) der tiefsten $T=1$-Zustände der selbstkonjugierten Kerne $(Z=N=A / 2)$ gilt:

$$
\Delta_{10}^{\text {experimentell }}-\Delta_{10}^{\text {berechnet }} \approx\left\{\begin{array}{cl}
E_{4}=60 \mathrm{keV} & \text { für } A=4 n+2 \\
0 & \text { für } A=4 n .
\end{array}\right.
$$

Dieses Ergebnis bestätigt die bereits in Fig. 4 dargestellte Aussage.

WILKINSON ${ }^{20}$ hat eine Reihe von physikalischen Gründen angegeben, die zu Energieverschiebungen der betrachteten Zustände führen können.

1. Konfigurationsmischungen zwischen dem tiefsten $T=1-Z$ ustand und den angeregten $T=0$-Zuständen der betrachteten selbstkonjugierten Kerne können zu negativen Energieverschiebungen führen. Der Effekt sollte bei den leichtesten Kernen mit $A=4 n$ am ausgeprägtesten sein, da dort wegen des großen $\Delta_{\mathbf{l} 0}$ (vgl. Tabelle 1) die Dichte von $T=0$-Zuständen in der Nähe des tiefsten $T=1$-Zustandes am größten ist.

2. Wie Thomas ${ }^{23}$ gezeigt hat, ergibt sich eine negative Energie-

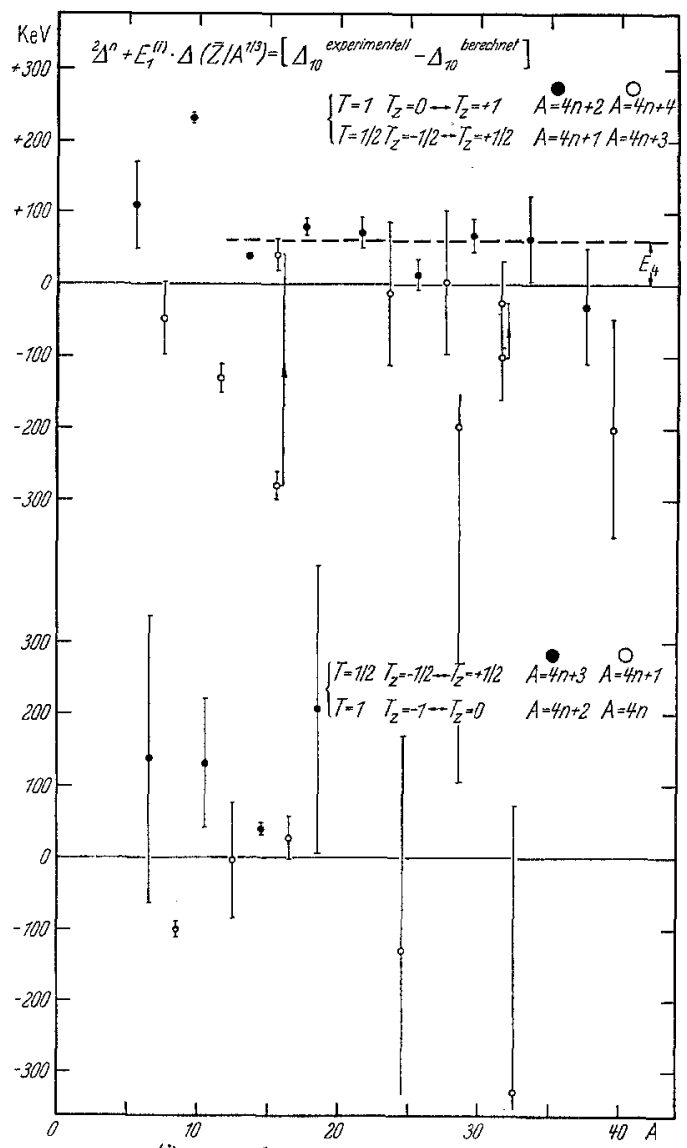

Fig. 5. $\left.{ }^{2} \Delta^{n}+E_{1}^{(i)} \cdot \Delta(\vec{Z}) A^{\frac{1}{3}}\right)=\left[\Delta_{10}\right.$ experimentell $-\Delta_{10}$ berechnet $]$ für die selbstkonjugierten Kerne als Funktion von $A$. (Vgl die entsprechenden Kurven in Fig. 2.) Bei $A=16$ und 32 wurden die Unstetigkeiten von ${ }^{1} \mathrm{~A}$ berïcksichtigt. Die Punkte zeigen eine positive Energieverschiebung der tiefsten $T=1$-Zustände der selbstkonjugierten Kerne mit $A=4 n+2$. Bei $A=4 n$ ist kein Effekt vorhanden verschiebung bei solchen Zuständen, die gegenüber Protonenemission instabil sind. Das fragliche Proton hält sich nämlich zeitweise am Rand des Kernes in einem Bereich kleinerer Coulomb-Energie auf. Der tiefste $T=1-Z$ ustand von $\mathrm{O}^{16}$ ist protoneninstabil, die entsprechenden Zustände

23 Thomas, R. G.: Phys. Rev. 88, 1109 (1952). 
von $\mathrm{Be}^{8}$ und $\mathrm{C}^{12}$ sind gerade noch protonenstabil. Auch dieser Effekt sollte also bevorzugt bei den leichtesten Kernen mit $A=4 n$ auftreten.

3. Es gibt eine Wechselbeziehung zwischen Coulomb-Kräften und Kernkräften. Die Coulomb-Kräfte führen zu einer Vergrößerung des Radius und ändern dadurch die Bindungsenergie. Dieser Effekt kann nach Wilkinson zu einer positiven Energieverschiebung führen.

4. Neben der Coulomb-Paarungsenergie abgesättigter Protonenpaare kann zusätzlich auch die Paarung einer geraden Anzahl von Nukleonen über die oben besprochene Wechselbeziehung zwischen CoulombKräften und Kernkräften zu Energieverschiebungen führen. Dieser Effekt sollte sich für $g g$ - und $u u$-Kerne, d.h. für die Kerne mit $A=4 n$ und $A=4 n+2$ verschieden verhalten.

Es läßt sich noch nicht entscheiden, ob einer dieser von WiLkinson diskutierten Effekte zur Deutung der gefundenen Energieverschiebung um etwa $60 \mathrm{keV}$ herangezogen werden kann. Der zuletzt angedeutete Effekt scheint am ehesten geeignet das Verhalten zu beschreiben, nämlich das Vorhandensein bei allen selbstkonjugierten $u$-Kernen, die Abwesenheit bei den $g g$-Kernen sowie die Unabhängigkeit von $\Delta_{10}$.

Nach der Coulomb-Paarungsenergie und der Energieverschiebung bei selbstkonjugierten Kernen soll nun versucht werden, die Energien $E_{1}^{(i)}$ und $E_{2}^{(i)}$ aus Gl. (5) zu deuten. In Anlehnung an Gl. (3) kann man für die Coulomb-Energien folgenden Ansatz machen:

$$
E_{c}(\text { i. Schale })=\frac{3}{5} \frac{e^{2}}{y_{0}^{(i)}} \frac{Z^{2}}{A^{\frac{1}{3}}}-Z \cdot S_{p}-\varepsilon_{1}^{(i)}-Z^{\prime} \cdot \varepsilon_{2}^{(i)}+\left[\frac{Z^{\prime}}{2}\right] \varepsilon_{3} .
$$

Die $Z$-Protonen werden dabei auf einen Kern (core) mit $Z-Z^{\prime}$-Protonen und auf $Z^{\prime}$-Außenprotonen aufgeteilt. An Stelle des für alle Protonen charakteristischen mittleren $r_{0}(A)$ aus Gl. (3) wird ein für die jeweiligen Außenprotonen charakteristisches $\gamma_{0}^{(i)}$ benutzt. Dann sind drei Korrekturglieder zu berücksichtigen. $\varepsilon_{1}^{(i)}$ beschreibt den für den Kern (core) gemachten Fehler, $\varepsilon_{2}^{(i)}$ beschreibt den für die Coulomb-Wechselwirkung zwischen dem Kern (core) und einem Außenproton gemachten Fehler und $\varepsilon_{3}$ beschreibt den für ein abgesättigtes Paar von Außenprotonen gemachten Fehler (Coulomb-Paarungsenergie). $\left[Z^{\prime} / 2\right]$ ist die größte ganze Zahl $\leqq Z^{\prime} / 2 . \quad S_{p}$ ist die Coulomb-Selbstenergie ${ }^{5,8,10,15,18}$ des Protons (Austauschterm). Durch Differenzbildung erhält man dann

$$
{ }^{1} \Delta(\text { i. Schale })=1,2 \cdot \frac{e^{2}}{\gamma_{0}^{(i)}} \cdot \frac{\bar{Z}}{A^{\frac{1}{3}}}-\left(\varepsilon_{2}^{(i)}+S_{p}\right)+\delta \cdot \varepsilon_{3} .
$$

Mit Ausnahme des fehlenden Gliedes $\delta^{\prime} \cdot E_{4}$ ist Gl. (9) mit Gl. (5) identisch, wenn man setzt:

$$
\begin{gathered}
E_{1}^{(i)}=1,2 \cdot \frac{e^{2}}{\gamma_{0}^{(i)}}, \\
E_{2}^{(i)}=\varepsilon_{2}^{(i)}+S_{p}, \\
E_{3}=\varepsilon_{3} .
\end{gathered}
$$


Die Werte für $E_{1}^{(i)}$ und $E_{2}^{(i)}$ in Tabelle 2 sowie Fig. 1 und Fig. 3 zeigen, da $B$ bei den Hauptschalenabschlïssen bei $A=4,16$ und 40 sowie beim Unterschalenabschlu $\beta$ bei $A=32$ Unstetigkeiten vorhanden sind. Es ist auffällig, da $B$ bei den anderen Unterschalenabschlüssen bei $A=12$ und 28 praktisch keine Sprünge zu erkennen sind.

Die Radien $r_{0}^{(i)}$ nach Gl. (10a) sind in Tabelle 2, Spalte 4 aufgeführt. Die Werte sind von vernünftiger Größe. Ein Vergleich von Gl. (3) mit Gl. (5) gibt die Möglichkeit, $r_{0}(A)$ als Funktion von $r_{0}^{(i)}$ (oder $E_{1}^{(i)}$ ), $\bar{Z} / A^{\hat{s}}, E_{2}^{(i)}, S_{p}, E_{3}$ und $E_{4}$ anzugeben.

$E_{2}^{\left(s \frac{b}{2}\right)}$ ist nach Gl. $(10 \mathrm{~b})$ zu deuten als Coulomb-Selbstenergie $S_{p}$ des Protons, da in dieser Schale $\varepsilon_{2}^{\left(s \frac{1}{2}\right)}=0$ ist. Unser Wert von $0,60 \mathrm{MeV}$ aus Tabelle 2, Spalte 3 ist in guter Übereinstimmung mit dem von CHERRY ${ }^{18}$ theoretisch gefundenen Wert von $0,58 \mathrm{MeV}$ für die CoulombSelbstenergie des Protons.

Wie bereits erwähnt, weichen einige experimentelle CoulombEnergiedifferenzen von Gl. (5) ab. Dies sind zunächst einige Spiegelkernwerte in der $p \frac{3}{2}$-Schale. Das reguläre Verhalten der Isobarentripletts in diesem Bereich läßt vermuten, daß sich bei diesen Spiegelkernen zusätzliche Effekte überlagern. Die große Abweichung der Coulomb-Energiedifferenz von $\mathrm{B}^{9}-\mathrm{Be}^{9}$ nach unten führt WILKINSON ${ }^{20}$ auf die bereits besprochene Thomas-Verschiebung ${ }^{23}$ zurück. $\mathrm{B}^{9}$ ist protoneninstabil und kann deshalb eine kleinere Coulomb-Energie besitzen. Es ist jedoch fraglich, ob dieser Effekt die vorhandenen Abweichungen erklären kann. Es müßten nämlich wegen der Instabilität von $\mathrm{Be}^{6}, \mathrm{~F}^{16}, \mathrm{O}^{16 *}, \mathrm{Li}^{5}$ und $\mathrm{He}^{5}$ auch Einflüsse auf die Coulomb-Energiedifferenzen bei $\mathrm{Be}^{6}-\mathrm{Li}^{6}, \mathrm{~F}^{16}-\mathrm{O}^{16 *}-\mathrm{N}^{16}$ und eventuell $\mathrm{Li}^{5}-\mathrm{He}^{5}$ vorhanden sein.

YAMAMoto und SteIgerT ${ }^{24}$ vermuten bei $\mathrm{P}^{30}$ einen zusätzlichen $T=1$-Zustand $0,26 \mathrm{MeV}$ unterhalb des bekannten $T=0$-Grundzustandes. Dies würde zu starken Abweichungen zwischen den zugehörigen CoulombEnergiedifferenzen und G1. (5) führen. In Tabelle 1 und Fig. 1 wurde der bisher bekannte tiefste $T=1$-Zustand mit $A_{10}=0,69 \mathrm{MeV}$ benutzt. Die Übereinstimmung mit G1. (5) ist gut. Bei einer Inversion der tiefsten $T=1$ - und $T=0-Z$ ustände müßte auch $\mathrm{P}^{30}$ ähnlich wie $\mathrm{Cl}^{34}$ einen übererlaubten $\beta^{+}$-Zerfall machen.

Der Wert für die Coulomb-Energiedifferenz von $\mathrm{Sc}^{41}-\mathrm{Ca}^{41}$ basiert im wesentlichen ${ }^{25}$ auf dem $Q$-Wert der Reaktion $\mathrm{Ca}^{40}(d, n) \mathrm{Sc}^{41}$. Die Werte für ${ }^{1} \Delta$ in Tabelle $1 \mathrm{bzw}$. die beiden in Fig. 1 und 3 angegebenen Punkte beziehen sich auf $Q$-Werte von $-0,57 \pm 0,05 \mathrm{MeV}$ bzw. $-1,13 \pm$ $0,05 \mathrm{MeV}$. Plende und Steigert ${ }^{25}$ betrachten den ersten Wert als

24 Yamamoto, S.S., u. F.E. Steigert: Phys. Rev. 117, 535 (1960).

25 Plendi, H.S., u. F.E. Steigert: Phys. Rev. 116, 1534 (1959). 
Q-Wert für den Übergang in den Grundzustand, Gl. (5) macht den zweiten Wert wahrscheinlicher ${ }^{25}$ a.

Es ist erstaunlich, da $\beta$ das Gesamtverhalten der Coulomb-Energiedifferenzen mit nur geringfügigen Abweichungen durch die einfache Gl. (5) beschrieben werden kann. Die Werte $E_{1}^{(i)}, E_{2}^{(i)}, E_{3}$ und $E_{4}$ in Gl. (5), Gl. (6) und Tabelle 2 sind jedoch rein empirisch ermittelt. Eine Theorie für die Coulomb-Energie einzelner Kerne oder für das allgemeine Verhalten aller betrachteten Kerne müßte in der Lage sein, auf Grund von Voraussetzungen über die Kopplungen zwischen den Nukleonen, über die Potentiale, Konfigurationsmischungen etc. diese Werte $\mathrm{zu}$ berechnen. Beispielsweise müßte auch das unterschiedliche Verhalten bei den Abschlüssen der Unterschalen bei $A=12,28$ und 32 erklärbar sein und damit Aufschlüsse über die Art der Kopplung zwischen den Nukleonen geben. Die theoretischen Arbeiten, die sich mit den Coulomb-Energiedifferenzen insbesondere zwischen den Spiegelkernen befassen, beschreiben das allgemeine Verhalten mit Sprüngen an den Schalenabschlüssen und die überlagerten Oszillationen; eine quantitative Übereinstimmung ist aber noch nicht vorhanden.

Herrn Dr. C. MAYER-BöRICKE vom Max-Planck-Institut für Kernphysik, Heidelberg sowie den Herren Dr. H.J. MANG und Dipl.-Phys. E. MaschKE vom Institut für Theoretische Physik, Heidelberg, bin ich für eingehende Diskussionen sehr zu Dank verpflichtet.

\section{Anhang}

Gl. (5) mit Gl. (6) und Tabelle 2 gestattet Voraussagen über noch nicht oder nur ungenau gemessene Werte. Die Tabellen 4, 5 und 6 bringen die berechneten Werte für den Energieunterschied zwischen den tiefsten Zuständen zu $T=1$ und $T=0$ bei selbstkonjugierten Kernen, für die Massendefekte $(M-A)$ einiger Kerne sowie für die maximalen $\beta^{+}$-Energien und Halbwertszeiten einiger übererlaubter $\mathrm{O}^{+} \rightarrow \mathrm{O}^{+}$-Fermi-Übergänge. Bei der Berechnung ${ }^{26}$ der (partiellen) Halbwertszeiten dieser $\beta$-Übergänge wurde der $f t$-Wert des Zerfalls von $\mathrm{O}^{\mathbf{1 4}}$ benutzt ${ }^{\mathbf{2 7}}$.

25 a Bemerkung bei der Korrektur. Die Diskrepanz zwischen der bisher bekannten Coulomb-Energiedifferenz von $\mathrm{Sc}^{41} \mathrm{Ca}^{41}$ und $\mathrm{Gl}$. (5) ist durch neuere Messungen praktisch beseitigt. Für ${ }^{1} \Delta$ ergibt sich:

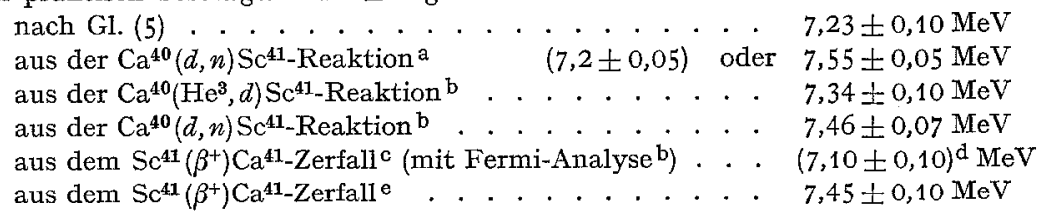

a) Plendr, H.S., u. F.E. Steigert: Private Mitteilung.

b) WEgENER, H.E., and W.S. HALL: Phys. Rev, wird veröffentlicht. - Bull. Amer. Phys. Soc. 5, 248 (1960).

c) Elirot, D.R., and L. D.P. KING: Phys. Rev. 60, 489 (1941).

d) Dieser Wert ist vermutlich durch Beimengung von $K^{38}$ erniedrigt. Vg1.

JÄNECKE, J.: Z. Naturforsch. 14a, 593 (1960).

e) Class, FARMer and Cramer: Phys. Rev., wird veröffentlicht.

${ }_{26}$ Moszxowski, S.A., u. K.M. Jantzen: UCLA Techn. Report 10-26-55.

27 Kistner, O.C., u. B.M. Rustad: Phys. Rev. 114, 1329 (1959). 
Tabelle 4. Berechnete Werie für den Energieunterschied $\Delta_{10}$ zwischen den tiefsten Zuständen zu $T=1$ und $T=0$

\begin{tabular}{c|c|c|c}
\hline $\begin{array}{c}(1) \\
\mathrm{Kera}\end{array}$ & $\begin{array}{c}(2) \\
\Delta_{\mathbf{1 0}} \text { in MeV }\end{array}$ & $\begin{array}{c}(1) \\
\mathrm{Kern}\end{array}$ & $\begin{array}{c}(2) \\
\Delta_{10} \text { in } \mathrm{MeV}\end{array}$ \\
\hline $\mathrm{Ne}^{20}$ & $10,22 \pm 0,10$ & $\mathrm{Cr}^{48}$ & $(6,0)$ \\
$\mathrm{Ar}^{36}$ & $6,60 \pm 0,10$ & $\mathrm{Fe}^{52}$ & $(6,2)$ \\
$\mathrm{Ca}^{40}$ & $7,50 \pm 0,10$ & $\mathrm{Ni}^{56}$ & $(5,9)$ \\
$\mathrm{Ti}^{44}$ & $(6,6)$ & &
\end{tabular}

Tabelle 5. Berechnete Werte für. den Massendefekt einiger Kerne

\begin{tabular}{l|c|c|c|c|c}
\hline $\begin{array}{c}(1) \\
\operatorname{Kern}\end{array}$ & $\begin{array}{c}(2) \\
(M-A) \text { in } \mathrm{MeV}\end{array}$ & $\begin{array}{c}(1) \\
\operatorname{Kern}\end{array}$ & $\begin{array}{c}(2) \\
(M-A) \text { in } \mathrm{MeV}\end{array}$ & $\begin{array}{c}(1) \\
\text { Kern }\end{array}$ & $\begin{array}{c}(2) \\
(M-A) \mathrm{in} \mathrm{MeV}\end{array}$ \\
\hline $\mathrm{Sc}^{41}$ & $-16,57 \pm 0,10$ & $\mathrm{~S}^{30}$ & $-5,14 \pm 0,10$ & $\mathrm{~V}^{\mathbf{4 4}}$ & $-10,89 \pm 0,20$ \\
$\mathrm{Ti}^{43}$ & $-16,62 \pm 0,10$ & $\mathrm{Cl}^{32}$ & $-3,94 \pm 0,10$ & $\mathrm{Cr}^{46}$ & $-16,24 \pm 0,20$ \\
$\mathrm{Ne}^{18}$ & $10,59 \pm 0,10$ & $\mathrm{Ar}^{34}$ & $-8,45 \pm 0,10$ & $\mathrm{Mn}^{48}$ & $-15,18 \pm 0,20$ \\
$\mathrm{Na}^{20}$ & $12,72 \pm 0,10$ & $\mathrm{~K}^{36}$ & $-6,81 \pm 0,10$ & $\mathrm{Fe}^{50}$ & $-20,01 \pm 0,30$ \\
$\mathrm{Mg}^{22}$ & $6,05 \pm 0,10$ & $\mathrm{Ca}^{38}$ & $-10,92 \pm 0,10$ & $\mathrm{Co}^{52}$ & $-19,04 \pm 0,30$ \\
$\mathrm{Al}^{24}$ & $7,00 \pm 0,10$ & $\mathrm{Sc}^{40}$ & $-9,00 \pm 0,10$ & $\mathrm{Ni}^{54}$ & $-23,52 \pm 0,30$ \\
$\mathrm{Si}^{26}$ & $0,49 \pm 0,10$ & $\mathrm{Ti}^{\mathbf{4 2}}$ & $-12,80 \pm 0,20$ & $\mathrm{Cu}^{56}$ & $-22,06 \pm 0,30$ \\
$\mathrm{P}^{28}$ & $1,24 \pm 0,10$ & & & &
\end{tabular}

Tabelle 6. Berechnete Werte für die maximalen $\beta$-Energien und partiellen Halbwertszeiten einiger übererlaubter $\mathrm{O}^{+} \rightarrow \mathrm{O}^{+}$-Positronenübergänge

\begin{tabular}{|c|c|c|c|c|}
\hline $\begin{array}{c}\text { (1) } \\
\text { Übergang }\end{array}$ & $\begin{array}{c}\text { (2) } \\
E_{\beta}^{+} \text {in } \mathrm{MeV} \\
\text { berechnet }\end{array}$ & $\begin{array}{c}\text { (3) } \\
E_{\beta}+\text { in } \mathrm{MeV} \\
\text { experimentell }\end{array}$ & $\begin{array}{c}\text { (4) } \\
T_{\frac{1}{2}} \text { in sec } \\
\text { berechnet }\end{array}$ & $\begin{array}{c}\text { (5) } \\
T_{\frac{1}{2}} \text { in sec } \\
\text { experimentell }\end{array}$ \\
\hline $\begin{aligned} & \mathrm{Ne}^{18} \rightarrow \mathrm{F}^{18 *} \\
& \rightarrow \mathrm{F}^{18} \\
& \mathrm{Mg}^{22} \rightarrow \mathrm{Na}^{22 m} \mathrm{Si}^{26} \rightarrow \mathrm{Al}^{26 m} \\
& \mathrm{~S}^{30} \rightarrow \mathrm{P}^{30 *} \\
& \mathrm{Ar}^{34} \rightarrow \mathrm{Cl}^{34} \\
& \mathrm{Ca}^{38} \rightarrow \mathrm{K}^{38 m} \\
& \mathrm{Sc}^{42} \rightarrow \mathrm{Ca}^{42} \\
& \mathrm{Ti}^{42} \rightarrow \mathrm{Sc}^{42} \\
& \mathrm{Cr}^{46} \rightarrow \mathrm{V}^{46} \\
& \mathrm{Fe}^{50} \rightarrow \mathrm{Mn}^{50} \\
& \mathrm{Ni}^{54} \rightarrow \mathrm{Co}^{54}\end{aligned}$ & $\begin{array}{l}2,29 \pm 0,10^{a} \\
3,37 \pm 0,10^{\mathrm{b}} \\
3,06 \pm 0,10 \\
3,78 \pm 0,10 \\
4,46 \pm 0,10 \\
4,94 \pm 0,10 \\
5,49 \pm 0,10 \\
5,41 \pm 0,10 \\
5,72 \pm 0,10 \\
6,47 \pm 0,10 \\
7,05 \pm 0,10 \\
7,62 \pm 0,10\end{array}$ & $3,2 \pm 0,2^{b}$ & $\begin{array}{l}27,5^{\mathrm{a}} \\
\\
8,0 \\
3,2 \\
1,6 \\
1,0 \\
0,64 \\
0,70 \\
0,54 \\
0,30 \\
0,20 \\
0,14\end{array}$ & $\begin{array}{l}1,6 \pm 0,2^{\mathrm{b}} \\
1,7^{\mathrm{c}} \\
0,66 \pm 0,05^{\mathrm{d}} \\
0,689 \pm 0,008^{\hat{1}} \\
1,1^{\mathrm{c}}\end{array}$ \\
\hline
\end{tabular}

a) Etwa $5 \%$ aller Zerfälle sollten einen übererlaubten Übergang mit einer partiellen Halbwertszeit von etwa $27,5 \mathrm{sec}$ in den ersten angeregten Zustand von $\mathrm{F}^{18}$ mit $T=1$ machen.

b) Der erlaubte Übergang in den Grundzustand von $\mathrm{F}^{18}$ hat einen sehr kleinen $f t$-Wert ähnlich wie beim Ửbergang $\mathrm{He}^{6} \rightarrow \mathrm{Li}^{6}$. Gow, J.D., u. L.W. Alvarez: Phys. Rev. 94, 365 (1954).

c) Tyren, H., u. P.A. Tove: Phys. Rev. 96, 773 (1954).

d) Cline, J.E., u. P.R. Chagnon: Phys. Rev. 108, 1495 (1957).

e) Cioutier, J.A.R., u. A. Hendrikson: Canad. J. Phys. 35, 1190 (1957).

f) J ̈̈NECKE, J.: Z. Naturforsch. 14a, 593 (1960).

Z. Physik. Bd. 160 